\title{
Implementing mHealth Apps for Child Developmental Screenings: Opportunities and Challenges
}

Andrew Miller ${ }^{1}$, PhD; Katie Swec ${ }^{2}$, MD; Sarah Watkins ${ }^{2}$; Elizabeth $\mathrm{Chen}^{2}$; Alison Paul ${ }^{2}$; Jordan Huskins ${ }^{2}$, MD; Jin Lee $^{3}, \mathrm{PhD}$; Nerissa Bauer ${ }^{2}, \mathrm{MD}$

${ }^{1}$ Department of Human-Centered Computing, School of Informatics and Computing, Indiana University-Purdue University Indianapolis, Indianapolis, IN, United States

${ }^{2}$ School of Medicine, Indiana University, Indianapolis, IN, United States

${ }^{3}$ BabyNoggin, San Francisco, CA, United States

\section{Corresponding Author:}

Andrew Miller, $\mathrm{PhD}$

Department of Human-Centered Computing

School of Informatics and Computing

Indiana University-Purdue University Indianapolis

535 W Michigan Street

Indianapolis, IN,

United States

Phone: 2787674

Email: andrewm@iupui.edu

\section{Abstract}

Background: Clinical care guidelines for universal developmental screening are meant to identify at-risk children as early as possible so that early intervention can be initiated. However, despite years of initiatives, developmental screening rates remain less than ideal. Recent innovations in mHealth apps that integrate developmental screening may offer an alternate way to optimize screening rates if it can encourage co-use and seamless integration into clinical workflow.

Objective: Prior to the planned deployment of a clinic-based mHealth developmental screening app, the research team sought to identify and characterize opportunities and challenges to implementation, focusing on clinic workflow and multi-stakeholder engagement with providers and parents.

Methods: Three clinic settings were recruited to participate from one children's hospital in Indianapolis, Indiana. Preimplementation clinical observations of workflow and team dynamics were performed. Potential adoption barriers and facilitators were explored through interviews with health care teams guided by the Consolidated Framework for Implementation Research (CFIR). Parents with children aged 0-5 were recruited from study clinics and social media to complete a 26-item survey to gauge their attitudes towards using apps in place of paper screening forms and for tracking their child's development.

Results: Proposed implementation workflows were co-created with each clinical team so to not increase overall visit length, which was the main concern for health care providers. Providers had enthusiasm for use of mHealth tools; however, concerns were expressed about potential technology failures, data security and HIPAA and the workflow impacts. Two hundred fifty parents responded to the social media survey. The top reason for downloading child health apps were for a convenient way to track development (62.6\%). Two-thirds of respondents expressed interest in an app that included developmental screening forms. Most parents prefer to complete screening tools on the day of the clinic visit (47.7\%) or electronically prior to the visit (44.8\%). Seventy-four percent of parents expressed a higher likelihood of using an app if recommended by the pediatrician.

Conclusions: Parents and providers are interested and open to mHealth apps for child developmental screening. Provider buy-in and involvement in implementation planning is critical, both to integrating apps into clinic workflows and to encouraging parents to use the app.

(iproc 2018;4(2):e11787) doi: 10.2196/11787

\section{KEYWORDS}

mobile health; mHealth; pediatrics; screening 
Edited by T Hale; this is a non-peer-reviewed article. Submitted 02.08.18; accepted 29.08.18; published 17.09.18.

Please cite as:

Miller A, Swec K, Watkins S, Chen E, Paul A, Huskins J, Lee J, Bauer N

Implementing mHealth Apps for Child Developmental Screenings: Opportunities and Challenges

iproc 2018;4(2):e11787

URL: http://www.iproc.org/2018/2/e11787/

doi: $\underline{10.2196 / 11787}$

PMID:

(C)Andrew Miller, Katie Swec, Sarah Watkins, Elizabeth Chen, Alison Paul, Jordan Huskins, Jin Lee, Nerissa Bauer. Originally published in Iproceedings (http://www.iproc.org), 17.09.2018. This is an open-access article distributed under the terms of the Creative Commons Attribution License (https://creativecommons.org/licenses/by/4.0/), which permits unrestricted use, distribution, and reproduction in any medium, provided the original work, first published in Iproceedings, is properly cited. The complete bibliographic information, a link to the original publication on http://www.iproc.org/, as well as this copyright and license information must be included. 